\title{
Which patient is going to benefit from surgical cytoreduction with intraperitoneal chemotherapy (for manifest peritoneal metastases)?
}

\author{
Sebastian Blaj, Pompiliu Piso
}

Klinik für Allgemein- und Viszeralchirurgie, Krankenhaus Barmherzige Brüder Regensburg, Prüfeninger 86, 93049 Regensburg, Germany

\begin{tabular}{|c|c|}
\hline ARTICLE INFO & ABSTRACT \\
\hline $\begin{array}{l}\text { Received } 18 \text { December } 2017 \\
\text { Accepted } 29 \text { December } 2017\end{array}$ & $\begin{array}{l}\text { The gastric cancer with peritoneal metastases still represents a serious problem in the } \\
\text { oncological surgery, whereas the peritoneal metastases arising from colorectal cancer }\end{array}$ \\
\hline $\begin{array}{l}\text { Keywords: } \\
\text { peritoneal neoplasms, } \\
\text { hyperthermia, } \\
\text { induced, } \\
\text { stomach neoplasms, } \\
\text { cytoreduction surgical } \\
\text { procedures, } \\
\text { induction chemotherapy, } \\
\text { laparoscopy. }\end{array}$ & $\begin{array}{l}\text { can be successfully treated using a multimodal therapy concept consisting of systemic } \\
\text { chemotherapy, cytoreductive surgery and hyperthermic intraperitoneal chemotherapy. } \\
\text { The best survival rates in patients suffering from peritoneal metastases of gastric cancer } \\
\text { have been reported by the ToGa trial, in patients with HER-2 positive expression who } \\
\text { received standard chemotherapy and trastuzumab. Cytoreductive surgery and HIPEC are } \\
\text { increasingly wining a place in the multimodal therapy of gastric cancer with peritoneal } \\
\text { metastases, the criteria for such an aggressive therapy are strict and the operability needs } \\
\text { to be previously assessed by laparoscopic exploration. Interesting new data are awaited } \\
\text { from the German GASTRIPEC study and from the French GASTRICHIP study. }\end{array}$ \\
\hline
\end{tabular}

\section{INTRODUCTION}

The peritoneal carcinomatosis of digestive and nondigestive origin was considered for a long time a final and incurable condition. In the beginning of the 1990's, the first publications on the multimodal treatment concept (cytoreductive surgery and intraperitoneal chemotherapy) led to a paradigm change in this field. To this date, as a result of several clinical studies, many Western European countries (e.g., Great Britain, Germany, France and Italy) have introduced in their guidelines, a multimodal therapy consisting of cytoreductive surgery and hyperthermic intraperitoneal chemotherapy as the standard of treatment for the patients with peritoneal carcinomatosis of colo-rectal origin. In well selected patients (low Peritoneal Cancer Index, good performance status, no extraabdominal metastases, no more than three peripheral good resectable liver metastases, no tumour-related biliary and / or ureteral obstruction, well / moderate differentiated histology, no signs of progress under systemic chemotherapy), a five-year survival of up to $50 \%$ can be achieved, provided the patients are treated in experienced centres [1].

Based on the good evidence and experience with the colorectal carcinomatosis, an extrapolation of this therapy concept to the patients with peritoneal carcinomatosis of

\footnotetext{
* Corresponding author

e-mail: pompiliu.piso@barmherzige-regensburg.de
}

gastric origin may be conceivable, but because of the different tumour biology, more restrictive selection criteria may be needed. However, peritoneal metastases are more frequent than in colorectal cancer and the peritoneum is still the main site of recurrence for gastric cancer.

\section{SYSTEMIC CHEMOTHERAPY FOR PATIENTS WITH GASTRIC CANCER AND PERITONEAL METASTASES}

To this date, there are no published trials investigating the effect of systemic chemotherapy in patients with gastric cancer and isolated peritoneal metastases. The core of the palliative systemic therapy is - worldwide - represented by a combination of platinum compounds and a fluorpyrimidine. In addition to that, a third drug (docetaxel or epirubicine) may be added. Best survival data provided by the ToGa trial shows a significant better survival rate in patients receiving trastuzumab in addition to systemic chemotherapy, as compared to patients who received systemic chemotherapy alone (median overall survival - 13.8 months $(95 \%$ CI 12-16) versus - 11.1 months (10-13) HR 0.74 ; $95 \%$ CI 0.60 $0.91, \mathrm{p}=0.0046))$. However, the data of the ToGa trial may be applied only in patients with a strong HER-2 expression (FISH positive or a score $3+$ in the immunohistochemistry) - which is a rather small group of patients [2]. 
Recently published data from the German FLOT study group showed that the patients with limited metastases of gastric cancer or cancer of the eso-gastric junction who received neaodjuvant chemotherapy and were subsequently operated upon had a more favourable outcome. The patients were included into three groups: one group with resectable disease, the second with limited metastatic disease and the third with extensive metastatic disease. All the patients in the second and third group received pre- and postoperative, a systemic chemotherapy after the FLOT (5-FU, leucovorin, oxaliplatin and docetaxel) regimen, with a median of eight cycles (1-15 cycles). The patients with extensive metastatic disease were operated only in palliative intent and the group also included patients with peritoneal metastases. The median overall survival was 22.9 months for the patients with limited metastatic disease, as compared to 10.7 months (HR 0.37, 95\% CI 0.25-0.55, p < 0.001) for those with extensive metastatic disease [3].

\section{ASSESSMENT OF RESECTABILITY AND COMPLETENESS OF CYTOREDUCTION}

Because of the pattern of tumour spreading and its biology, a complete staging is of uttermost importance. Lesions which are smaller than $5 \mathrm{~mm}$ - cannot be seen either in the CT-scan or in MRI. Therefore, an exploratory laparoscopy may bring additional information, especially regarding the extent of the peritoneal carcinomatosis or the amount of small nodular tumour on the small bowel and its mesentery. In such cases and because the aim of any potentially curative surgery is to remove all macroscopic tumour, the diffuse involvement of the bowel and its mesentery represents a contraindication for a multimodal therapy, hence, a complete cytoreduction is technically not possible.

Paul Sugarbaker initially described the surgical technique of parietal and visceral peritonectomy [4]. Our group in Regensburg, Germany published in 2015, a detailed technique with an additional video [5].

\section{QUANTIFICATION OF THE EXTENT OF DISEASE WITH THE PERITONEAL CANCER INDEX (PCI)}

The extent of the peritoneal disease can be estimated using the semi-quantitative Peritoneal Cancer Index. In order to determine it, the abdomen is divided into nine equal squares and the small bowel into four regions (proximal and distal jejunum and ileum, respectively). Each lesion on the surface of the parietal and visceral peritoneum is given a score between zero and three according to its size. Therefore, it is possible to achieve a maximum PCI of 39 [6]. An estimation of the PCI via laparoscopy or CT-scan is possible, although not very sensitive; the real extent of the disease is often underestimated.

\section{HYPERTHERMIC INTRAPERITONEAL CHEMO- THERAPY (HIPEC) AS AN ADDITIVE LOCO- REGIONAL TREATMENT}

Even after a complete macroscopical cytoreduction, there are still free tumour cells in the peritoneal cavity that can lead to relapse. Therefore, a hyperthermic intraperitoneal chemotherapy may help, especially because the concentration of the chemotherapeutic agent that can be reached in the peritoneal cavity is much higher that after the systemic administration. Furthermore, the side effects after the intraperitoneal chemotherapy are not so pregnant as after the systemic chemotherapy. Ideal for an intraperitoneal chemotherapy are agents with a high molecular weight (e.g. cisplatin) and with a long retention time in the abdomen. The penetrance depth into the tumour nodules can be as high as $2-3 \mathrm{~mm}$ and it is enhanced by the temperature. The chemotherapeutic agents are applied using either the open or the closed abdomen technique. Several drains and temperature probes are placed into the abdomen and after reaching an intraperitoneal temperature of $42-43^{\circ} \mathrm{C}$, the chemotherapy is added. The carrier solution is mostly $5 \%$ dextrose (mainly for the platinum derivatives) or isotonic saline. The circulation of the heated solution takes between 30 and 90 minutes depending on the protocol.

\section{ASSOCIATED MORBIDITY AND MORTALITY}

The multimodal therapy represents a huge burden for the patient. Generally, every third patient develops a complication that requires a type of procedure (radiological intervention or re-do surgery) [7].

In the beginning years, anastomotic fistulas represented a serious problem, today - we are experiencing a decrease of the incidence of this complication. The most frequent complications are represented by fascial dehiscence, paralytic ileus, and intraabdominal infections. Surgical haemorrhage is noted in $<2 \%$ of the patients, and serious problems that are directly related to the intraabdominal chemotherapy, i.e. leukopenia or renal toxicity are noted in less than $5 \%$ of the patients. The mortality lays in experienced centres under $5 \%$, in some tertial referral centres - even under $2 \%$. This is due to a better complication management consisting mostly in a more "aggressive" and early indication for diagnostic and re-do surgery.

\section{LONG-TERM RESULTS}

A recent publication following of a group of 81 patients operated in referral centres in France within a period of 20 years showed an overall survival rate of $18 \%$. Herein, eleven percent of the patients were considered "cured", meaning that they were disease free after five years post-procedure. The majority of patients received a hyperthermic intraperitoneal chemotherapy with Mitomycin C for 90 minutes at $42^{\circ} \mathrm{C}$. All the patients who were disease-free after five years post-procedure had as independent factors in a multivariate analysis - a PCI $<7(\mathrm{p}=0.12)$, a synchronous peritoneal carcinomatosis $(\mathrm{p}=0.02)$ and a complete cytoreduction $(\mathrm{p}=0.09)$ [8].

A randomized phase III trial from China was designed to study the effects of the multimodal therapy (cytoreductive surgery and HIPEC) in patients with peritoneal carcinomatosis and gastric cancer. The two groups (only cytoreductive surgery and cytoreductive surgery plus HIPEC) showed a median survival of 6.5 months (95\% CI 4.8-8.2 months) and 
11.0 months (95\% CI 10.0-11.9 months), respectively. The multivariate analysis of the patients in the HIPEC group identified as independent factors for a better survival - the number of systemic therapy cycles $(>6)$, the synchronous peritoneal carcinomatosis, a complete cytoreduction, and the absence of serious adverse events [9].

The GYMSSA trial conducted in the National Cancer Institute, USA compared the multimodal therapy (cytoreductive surgery, HIPEC, and systemic chemotherapy with FOLFOXIRI) with the systemic chemotherapy alone (FOLFOXIRI) in patients with peritoneally metastasized gastric cancer. Despite the minimal patient collective (only sixteen patients were enrolled and randomized), the study showed a median overall survival of 11.3 months in the multimodal group and of only 4.3 months in the chemotherapy alone group. None of the patients who received chemotherapy alone survived beyond a year and all the patients who were alive after one year received a complete cytoreduction and had a low PCI [10].

The efficacy of a new multimodal approach in patients with peritoneal carcinomatosis and gastric cancer was evaluated in a single centre in Japan. According to the study protocol, the patients received a bidirectional systemic and intraperitoneal induction chemotherapy consisting of normothermic intraperitoneal docetaxel and cisplatin, followed by four cycles of S1 (tegafur/gimeracil/oteracil); only the patients who responded to the induction therapy were subsequently operated upon and received a hyperthermic intraperitoneal chemotherapy with docetaxel. The patients who received the multimodal therapy had a 5 -year survival of $10.7 \%$, the median survival rate was 15.8 months. The multivariate analysis identified as independent predictors for a better prognosis, the low PCI $(\leq 6, p=0.001)$, a good pathological response $(\mathrm{p}=0.001)$ and a complete cytoreduction $(\mathrm{p}=0.001)[11]$.

A retrospective matched-pairs analysis demonstrated that patients with peritoneal carcinomatosis and gastric cancer who received cytoreductive surgery and HIPEC have a better prognosis, as compared to those who received only gastrectomy and palliative systemic chemotherapy [12].

A recently published meta-analysis of randomized and non-randomized trials between 1985 and 2016, which compared HIPEC with the standard systemic chemotherapy in patients with peritoneal carcinomatosis and gastric cancer, showed a better overall survival in the HIPEC group. It also concluded that the patients who have only a positive peritoneal cytology and a limited lymphnode involvement presumably benefitted most from the multimodal approach [13].

The German GASTRIPEC trial (NCT02158988) is a phase III prospective multicenter study which aims to evaluate the outcome of the patients with peritoneal carcinomatosis and gastric cancer or cancer of the eso-gastric junction with and without HIPEC. The patients with a negative or an unknown HER-2 status receive the EOX protocol, the patients with a positive HER-2 status receive cisplatin, capecitabine and trastuzumab. The patients in the experimental arm are subsequently operated upon and receive a hyperthermic intraperitoneal chemotherapy with mitomycin $\mathrm{C}$ and cisplatin. The patients in the no-HIPEC arm receive only the systemic chemotherapy. First data is expected in 2020.

\section{NOW, WHICH PATIENT IS GOING TO BENEFIT FROM SURGICAL CYTOREDUCTION WITH INTRAPERITONEAL CHEMOTHERAPY (FOR MANIFEST PERITONEAL METASTASES)?}

According to the data available to this day, the patients with peritoneal carcinomatosis and gastric cancer are having the best results after cytoreductive surgery and HIPEC when the following criteria are met:

- synchronous peritoneal carcinomatosis,

- low PCI $(<6)$,

- good response after induction chemotherapy,

- laparoscopy demonstrating the limited disease, high probability of resectable disease and histological response after systemic chemotherapy,

- complete surgical cytoreduction achieved and combined with HIPEC.

\section{PERSPECTIVES}

Far better than treating manifest peritoneal metastases is to avoid them, as patients with $\mathrm{T} 3$ or $\mathrm{T} 4$ tumors or $\mathrm{N}$ positive status have an increased risk of metastases. Still, adding HIPEC to the oncological resection of the primary may reduce the risk of peritoneal metastases, as demonstrated in the past by several Asian studies. Yet information is still limited. Hence, a French phase III randomized, opened, multicentric clinical trial (GASTRICHIP, NCT01882933) aims to compare the 5-year overall survival in patients with advanced gastric cancer who received curative gastrectomy and HIPEC or only curative gastrectomy alone. First data is awaited May 2023.

\section{REFERENCES}

1. Elias D, Mariani A, Cloutier A, Blot F, Goéré D, Dumont F et al. Modified selection criteria for complete cytoreductive surgery plus HIPEC based on peritoneal cancer index and small bowel involvement for peritoneal carcinomatosis of colorectal origin. European Journal of Surgical Oncology (EJSO). 2014;40(11):1467-1473

2. Bang Y, Van Cutsem E, Feyereislova A, Chung H, Shen L, Sawaki A et al. Trastuzumab in combination with chemotherapy versus chemotherapy alone for treatment of HER2-positive advanced gastric or gastro-oesophageal junction cancer (ToGA): a phase 3, open-label, randomised controlled trial. The Lancet. 2010;376(9742):687-697.

3. Al-Batran S, Homann N, Pauligk C, Illerhaus G, Martens U, Stoehlmacher J et al. Effect of Neoadjuvant Chemotherapy Followed by Surgical Resection on Survival in Patients With Limited Metastatic Gastric or Gastroesophageal Junction Cancer. JAMA Oncology. 2017;3(9):1237.

4. Sugarbaker P. Peritonectomy Procedures. Annals of Surgery. 1995;221(1):29-42.

5. Piso P, Leebmann H, März L, Mayr M. Zytoreduktive Chirurgie für maligne Tumoren des Peritoneums. Der Chirurg. 2014;86(1):38-46.

6. Jacquet, P., Sugarbaker, P. H. Clinical research methodologies in diagnosis and staging of patients with peritoneal carcinomatosis. Cancer treatment and research. 1996;82, 359-374.

7. Chua T, Yan T, Saxena A, Morris D. Should the Treatment of Peritoneal Carcinomatosis by Cytoreductive Surgery and Hyperthermic Intraperitoneal Chemotherapy Still be Regarded as a Highly Morbid Procedure?. Annals of Surgery. 2009;249(6):900-907. 
8. Chia C, You B, Decullier E, Vaudoyer D, Lorimier G, Abboud $\mathrm{K}$ et al. Patients with Peritoneal Carcinomatosis from Gastric Cancer Treated with Cytoreductive Surgery and Hyperthermic Intraperitoneal Chemotherapy: Is Cure a Possibility?. Annals of Surgical Oncology. 2016;23(6):1971-1979.

9. Yang X, Huang C, Suo T, Mei L, Yang G, Cheng F et al. Cytoreductive Surgery and Hyperthermic Intraperitoneal Chemotherapy Improves Survival of Patients with Peritoneal Carcinomatosis from Gastric Cancer: Final Results of a Phase III Randomized Clinical Trial. Annals of Surgical Oncology. 2011;18(6):1575-1581.

10. Rudloff U, Langan R, Mullinax J, Beane J, Steinberg S, Beresnev T et al. Impact of maximal cytoreductive surgery plus regional heated intraperitoneal chemotherapy (HIPEC) on outcome of patients with peritoneal carcinomatosis of gastric origin: Results of the GYMSSA trial. Journal of Surgical Oncology. 2014;110(3):275-284.
11. Canbay E, Mizumoto A, Ichinose M, Ishibashi H, Sako S, Hirano M et al. Outcome Data of Patients with Peritoneal Carcinomatosis from Gastric Origin Treated by a Strategy of Bidirectional Chemotherapy Prior to Cytoreductive Surgery and Hyperthermic Intraperitoneal Chemotherapy in a Single Specialized Center in Japan. Annals of Surgical Oncology. 2013;21(4):1147-1152.

12. Boerner T, Graichen A, Jeiter T, Zemann F, Renner P, März L et al. CRS-HIPEC Prolongs Survival but is Not Curative for Patients with Peritoneal Carcinomatosis of Gastric Cancer. Annals of Surgical Oncology. 2016;23(12):3972-3977.

13. Desiderio J, Chao J, Melstrom L, Warner S, Tozzi F, Fong Y et al. The 30-year experience-A meta-analysis of randomised and highquality non-randomised studies of hyperthermic intraperitoneal chemotherapy in the treatment of gastric cancer. European Journal of Cancer. 2017;79:1-14. 